\title{
More food for thought
}

$\int \mathrm{r}$ ust before I started writing this editorial, the US media reported that federal food-safety officials were warning consumers to stop eating all foods that contain pistachios while investigators try to identify the source of a new salmonella outbreak. I thought, "nuts!"... we are still reeling from a 44-state (and Canadian) outbreak of salmonella infections linked to peanut products. This outbreak was traced to a single producer's peanut butter or peanut paste and affected more than 400 food products, which have subsequently been recalled.

According to a recent article (Maki, D. N. N. Engl. J. Med. 360, 949-953 [2009]), food-borne disease develops in 76 million Americans annually, causes 350,000 hospitalizations and 5,000 deaths and adds US $\$ 7$ billion to the cost of health care! In addition to direct costs and indirect costs secondary to loss-of-

productivity that are related to food-borne infections, upstream and downstream costs are involved. When a recent salmonella epidemic was erroneously attributed to contamination of tomatoes grown in the southwestern US, the tomato industry lost an estimated \$200 million. Ultimately, the outbreak was isolated to jalapeno and serrano peppers from a Mexican farm, which emphasizes the complexity of identifying contamination in our diets (as well as Americans' growing taste for salsa).

In an earlier editorial, I reviewed a cholera epidemic attributed to oysters eaten on an Alaskan cruise ship and linked to global warming. Numerous factors are influencing food safety as our food chain becomes increasingly complex and global. As Maki points out, "virtually all food consumed domestically is grown and processed on a vast industrial scale or, increasingly, is imported." We have watched from a distance the impact of the contamination of milk products with melamine in China that caused kidney disease in over 300,000 children. Food-related health risks are global, as decreasing amounts of the foods we eat are grown or produced locally and it would be impossible to feed the expanding population of the US or the world without efficient, industrialized food production. The challenge, as Maki states, is to improve the quality and safety of industrially produced foods. Furthermore, this problem is not unique to the US, as globalization has similar implications for food production and safety across the rest of the world.
Microbial contamination of food sources is not limited to manufactured food products but also affects meat, poultry, milk, eggs, and seafood. With centralized processing and distribution, contamination that occurs at any step in the food-production process can rapidly reach millions of consumers. Furthermore, ubiquitous use of antibiotics is leading to increasingly virulent and resistant strains of infectious agents and, as Maki explains, a "...weakening of animal's resistance to colonization by enteropathogens".

Numerous steps must be taken to improve the global production, processing and monitoring of food. Recognition of the substantial costs associated with food-borne illness will justify the commitments and expenditures by governments and their regulatory authorities to improve food safety. In addition to the reduction of unnecessary antibiotic use in human and veterinary medicine, Maki propones improved technologies, such as routine irradiation of final commercial products, which has not been uniformly accepted by the public in all venues despite its proven safety.

Microbial contamination is only one consideration regarding healthy foods and diets. Continued advocation of the benefits of omega-3 fatty acids, primarily through fish consumption, is contributing to the decline in worldwide fish stocks. Despite the comparatively low consumption of fish (averaging one serving per person per week) in the US, a recent article (Jenkins, X. et al. CMAJ 180, 633-637 [2009]) warns that adoption of the recommended intake of two servings of fatty fish per week would deplete the commercial fish stock within 40 years. Even enhanced fish farming is unlikely to resolve the reduction in available commercial seafood, resulting from inefficiencies related to the current practices of raising fish on fish meals and fish oils derived from smaller fish. In addition, considerable pollution related to fish farming exists that adversely affects the environment.

So, while finishing this editorial and devouring a pistachioless selection of nuts along with a smaller portion of shrimp on an airline flight, I continue to share concerns about global food safety and how we are going to feed healthy foods to an expanding worldwide population.

doi:10.1038/nrgastro.2009.66
Stephen B Hanauer is the Editor-in-Chief of Nature Reviews Gastroenterology \& Hepatology.

Competing interests The author declared no competing interests. 